\title{
Occlusion of retinal vessels using targeted delivery of a platelet aggregating agent
}

\author{
Yuichiro Ogura, Theresa Guran, Kanji Takahashi, Ran Zeimer
}

\begin{abstract}
Local laser targeted delivery of a platelet aggregating agent to occlude retinal and choroidal vessels was evaluated in rabbits and rats. Liposomes containing adenosine diphosphate (ADP) were administered intravenously and an argon laser was used to lyse the liposomes in main retinal arteries. Control vessels were treated with the same energy of laser without administering ADP. Fluorescein angiography performed 2 weeks later showed that all the control vessels were perfused. Ninety percent of the ADP-treated arteries showed complete or partial occlusion. Successful occlusion increased with the laser energy and decreased with increasing vessel diameter. Histopathology showed that occlusion was achieved in retinal as well as choroidal vessels. The inner retina remained relatively unaffected at the treatment site but the outer retina was thermally damaged. These preliminary results suggest that targeted delivery of a platelet aggregating agent holds promise for occluding vessels in the fundus.

(Brf Ophthalmol 1993; 77: 233-237)
\end{abstract}

Successful occlusion of the blood vessels without damaging adjacent healthy tissue is potentially applicable to treatment of various retinal and choroidal disorders such as tumours, vascular abnormality, diabetic retinopathy, and neovascular macular diseases, particularly agerelated macular degeneration. Photocoagulation using xenon and laser light has been studied to occlude blood vessels in the fundus. ${ }^{12}$ Photocoagulation, however, causes extensive damage to the adjacent tissues because of heat diffusion and is inefficient in occluding large vessels permanently. Cauterisation with a diathermy probe can be used to occlude retinal vessels but the procedure is invasive and also damages the adjacent tissues. ${ }^{3}$ Photodynamic therapy with photosensitising agents such as haematoporphyrin derivative, ${ }^{4}$ rose bengal, ${ }^{56}$ or chloroaluminium sulphonated phthalocyananine ${ }^{78}$ to reduce the required laser energy has been studied recently. These approaches, which reduce damage to surrounding tissue, have been unable to occlude large retinal vessels for 2 weeks or more.

We have developed a new method, laser targeted delivery, to deliver drugs locally into the retinal vasculature. ${ }^{910}$ Laser targeted delivery consists of encapsulating the photosensitising agent in temperature sensitive liposomes and administering them intravenously. The agent is then released at the target site in the retina by warming the liposomes to $41^{\circ} \mathrm{C}$ with a light pulse from a laser. Our previous studies suggested that a high concentration of a fluorescent dye can be released in the retinal vessels at the desired locations. ${ }^{10-12}$ Since the temperature increase required to lyse liposomes is only a few degrees, we suggest the possibility of causing intravascular thrombosis without damaging the adjacent retina. The purpose of this study is to evaluate whether laser targeted delivery is a promising method to occlude posterior pole vessels and to assess the efficacy of platelet aggregating agents for that purpose.

Adenosine diphosphate (ADP) is known to initiate aggregation of platelets in vitro as well as in vivo. ${ }^{13-16}$ Although platelet aggregation is rapidly reversible at low concentrations of ADP, high concentrations result in irreversible aggregation. ${ }^{15} 17$

\section{Materials and methods}

\section{LIPOSOME PREPARATION}

Adenosine diphosphate(ADP) (Fisher Scientific, Fair Lawn, NJ, USA) was encapsulated in temperature sensitive liposomes ${ }^{11}$ at a concentration of approximately $10^{-1} \mathrm{M}$. The liposomes were prepared by reverse phase evaporation and stored frozen in $2.5 \%$ mannose (Sigma, St Louis, MO, USA). Liposomes thus prepared appeared to be stable at $-20^{\circ} \mathrm{C}$ for more than 2 months. Liposomes were thawed immediately before use, then centrifuged and washed several times in sterile HEPES buffer with $2 \cdot 5 \%$ mannose to eliminate unencapsulated ADP from the suspension. These liposomes in serum have been shown to pass through a phase transition around $41^{\circ} \mathrm{C}$, at which point they release $80-90 \%$ of their contents. $^{9}$

\section{PLATELET AGGREGATING ACTIVITY}

The platelet aggregating activity of ADP was determined by adding $10 \mu \mathrm{l}$ of various dilutions of a stock ADP solution to $40 \mu$ l of fresh human platelet rich plasma. ${ }^{17}$ The mixture was rocked on a nutator for 6 minutes and then observed to determine at which dilutions large clumps of platelets were visible. The lowest concentration 


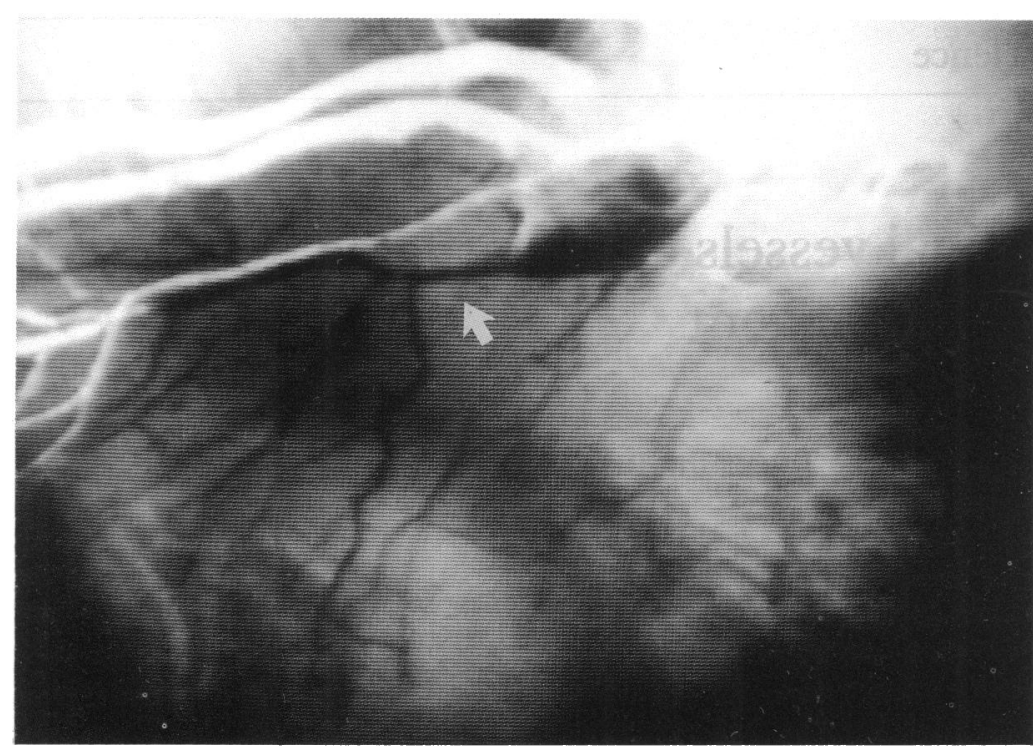

Figure 1 Fluorescein angiogram in rabbit 2 weeks after treatment with ADP liposomes. The treated vessel is closed (arrow).

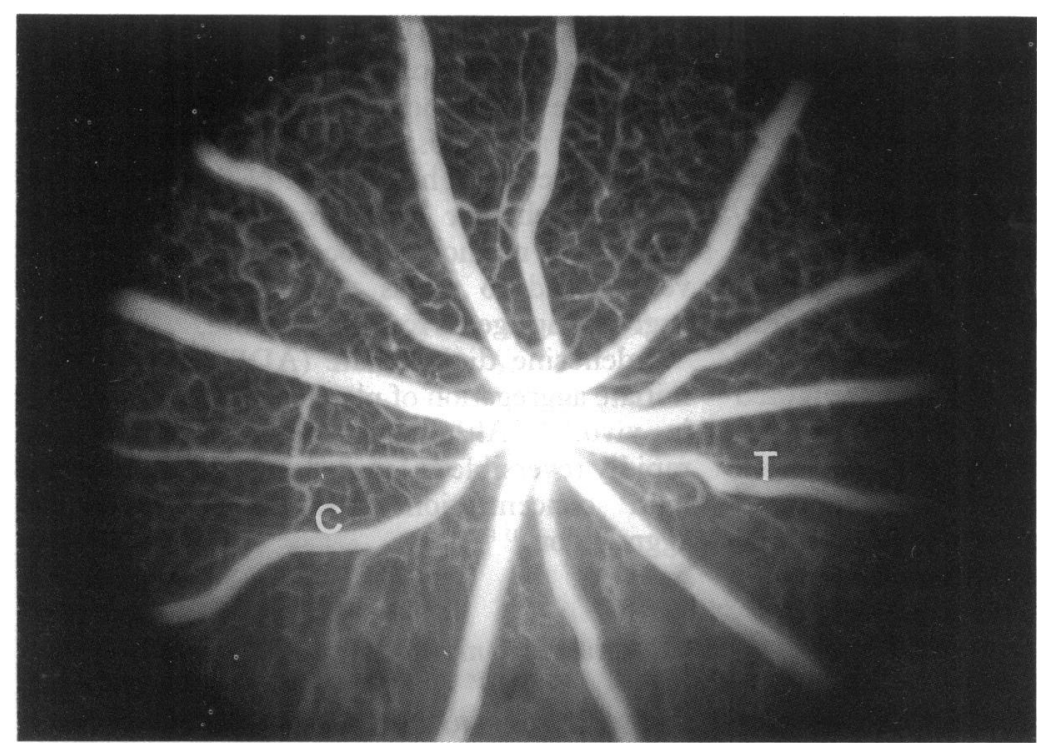

Figure $2(A)$ Fluorescein angiogram in rat before treatment. Control $(C)$ and treated $(T)$ vessels.

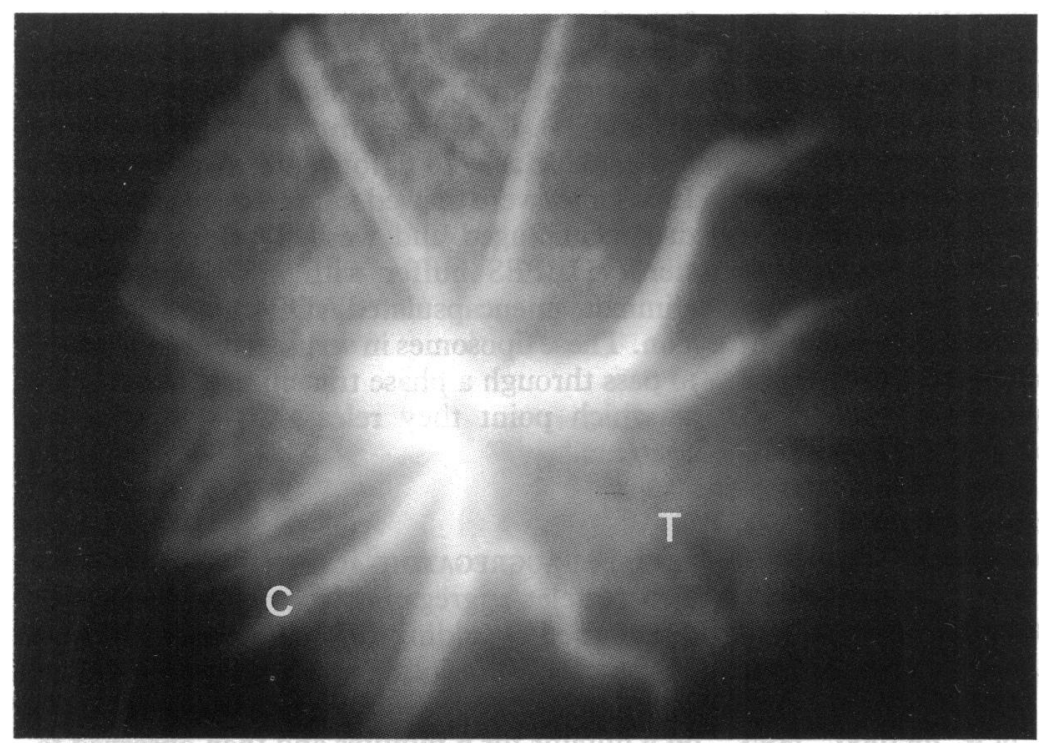

Figure 2(B) Fluorescein angiogram in rat 2 weeks after treatment. In the treated area $(T)$, not only is the target artery occluded, but the two adjacent veins are occluded as well. The control artery $(C)$ is reperfused. of ADP at which platelets formed large aggregates under these conditions was typically $5 \times 10^{-7} \mathrm{M}$. The concentration of unencapsulated ADP in liposome suspension was determined by making serial dilutions of the suspension and adding aliquots of these to the platelet rich plasma. The smallest dilution at which aggregates formed, when multiplied by the lowest concentration of ADP that formed aggregates, resulted in the concentration of unencapsulated ADP in liposomes. The concentration of free ADP in the resultant suspension was $10 \%$ or less of the encapsulated concentration.

To determine the total concentration of ADP in the suspension, the liposomes were first lysed using 5\% Triton X (Sigma, St Louis, MO) and then diluted. The rest of the procedure was analogous to that used in determining the concentration of unencapsulated ADP. All solutions were used within 2 hours of preparation for best results. Detergent, when diluted at the levels used in the assay, did not noticeably affect platelet aggregation.

\section{ANIMALS}

Seven Dutch-belted rabbits and 10 Long Evans hooded rats were used in compliance with the ARVO Resolutions on the Use of Animals in Research. The animals were anaesthetised with an intramuscular injection of a mixture of ketamine $\mathrm{HCl}(35 \mathrm{mg} / \mathrm{kg}$ for rabbits, $50 \mathrm{mg} / \mathrm{kg}$ for rats) and xylazine $(3.5 \mathrm{mg} / \mathrm{kg}$ for rabbits, $5 \mathrm{mg} / \mathrm{kg}$ for rats).

\section{TARGETED DELIVERY OF ADP}

The pupils were dilated with $1 \%$ tropicamide and $10 \%$ phenylephrine. After topical anaesthesia ( $0.5 \%$ proparacaine) was applied, a contact lens was placed on the cornea to visualise the fundus. The liposomes were injected intravenously in both animals with $5 \mathrm{mg}$ of $\mathrm{ADP} / \mathrm{kg}$ bodyweight. This dose provides a calculated blood concentration of encapsulated ADP of $5 \times 10^{-4} \mathrm{M}$. Liposomes were lysed locally in retinal vessels via a light pulse from an argon laser mounted on a slit-lamp biomicroscope (Carl Zeiss, Thornwood, NY, USA). The size of the laser spot was $800 \mu \mathrm{m}$ in diameter.

In experiments with rabbits, the laser was applied to a main retinal artery at powers of 200 $350 \mathrm{~mW}$. Laser pulses were applied until occlusion of the targeted vessel was observed. Ten arteries were treated with the ADP liposomes and three arteries (controls) were treated with the laser without administration of ADP liposomes.

The effect of the laser energy and the size of the vessels were evaluated in the experiments on rats. The laser was applied continuously for 5 minutes to arteries approximately two disc diameters away from the optic disc to cause constant release of the liposomes. Three power levels were used: 50,75 , and $100 \mathrm{~mW}$. A total of 21 arteries were treated with the ADP liposomes and 10 arteries (controls) received laser treatment alone. The diameters of the retinal arteries were determined from fluorescein angiograms 
obtained before treatment. Retinal vessel sizes were measured in rats from fluorescein angiograms using a densitometer. ${ }^{18}$ The calibration was corrected for the difference in axial length of the rat eye relative to that of a model of the human eye.

FOLLOW UP

Fluorescein angiograms were taken at 1 and 2 weeks to determine the results of treatment. All the results reported here are from 2 weeks after treatment.

Histopathological studies were performed on rats. The arteries were treated without (control) and after administration of ADP liposomes in the same procedure described above. Two eyes were enucleated immediately after treatment and another two eyes were enucleated 2 weeks after treatment. The enucleated eyes were fixed in a solution of $10 \%$ neutral buffered formalin. After the removal of the anterior segment, the eye cup was divided into the segments which included the laser lesions. Sections were prepared for light microscopy by standard methods and were stained by haematoxylin and eosin.

\section{Results}

\section{RABBIT STUDIES}

All three control vessels treated without ADP liposomes were reperfused at 2 weeks. However, nine vessels $(90 \%)$ treated with ADP liposomes showed occlusion on fluorescein angiography 2 weeks after treatment (Fig 1). The main arteries were partially reperfused in five eyes out of these nine, but branching arteries downstream were still closed.

\section{RAT STUDIES}

Examples of fluorescein angiograms taken before and 2 weeks after treatment are shown in Figure 2. The control vessel treated without ADP liposome administration was reperfused. The artery treated with ADP liposomes and the adjacent veins are shown occluded at 2 weeks.

The percentage of vessels occluded at 2 weeks depended on the laser power used during treatment, with more occlusions resulting from the use of higher powers (Fig 3). No vessels treated with a power of $50 \mathrm{~mW}$ showed occlusion, while $67 \%$ of the vessels treated with a power of $100 \mathrm{~mW}$ were occluded at 2 weeks. None of the control vessels showed occlusion at 2 weeks. The size of the vessels treated also played a role in the success rate of vessel occlusion (Fig 4). Smaller vessels were more easily occluded than larger ones.

Light photomicrographs of control and ADPtreated retinal lesions of the rat after the treatment are presented in Figures 5 and 6. Immediately after the treatment a thrombus was present in the control vessels, but the lumina were still partially patent (Fig 5A). The choriocapillaris and deeper choroidal vessels were occluded by thrombi consisting of fibrin, platelets, and erythrocytes. In the ADP-treated lesion, the targeted retinal vessels were occluded by

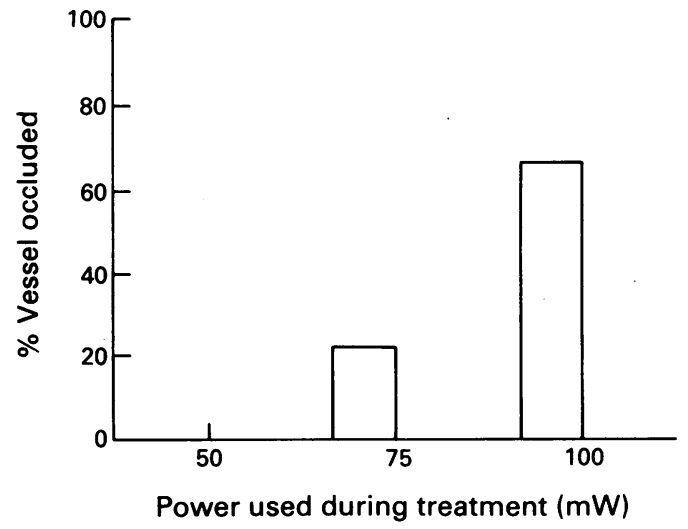

Figure 3 Effect of power used during treatment on the success rate of vessel occlusion at 2 weeks after treatment.

thrombi consisting of degenerated erythrocytes, platelets, and polymorphonuclear leucocytes (Fig 6A). The choriocapillaris and deeper choroidal vessels were also occluded by thrombi. In both control and treated lesions, the outer retinal layers including the photoreceptor, outer nuclear, outer plexiform, and inner nuclear layers were affected showing a granular appearance.

Two weeks after treatment the lumina of the control retinal vessels were recanalised, though the walls of the retinal vessels were thickened. The choriocapillaris and deeper choroidal vessels were also recanalised. The inner retinal layers were relatively well preserved with mild infiltration of pigment-laden macrophages, but there was a focal loss of photoreceptor cells (Fig 5B). In the ADP-treated lesion, the lumina of targeted retinal arteries narrowed and were occluded by thrombi consisting of fibrinous eosinophilic materials. In the choroid; the choriocapillaris and deeper choroidal vessels disappeared and round-shaped, pigment-laden macrophages migrated into the subretinal space and choroid. The retina, was markedly thinned with extensive loss of neuronal elements in full thickness of the retina, possibly due to severe retinal and choroidal ischaemia (Fig 6B).

\section{Discussion}

The results of this feasibility study indicate that

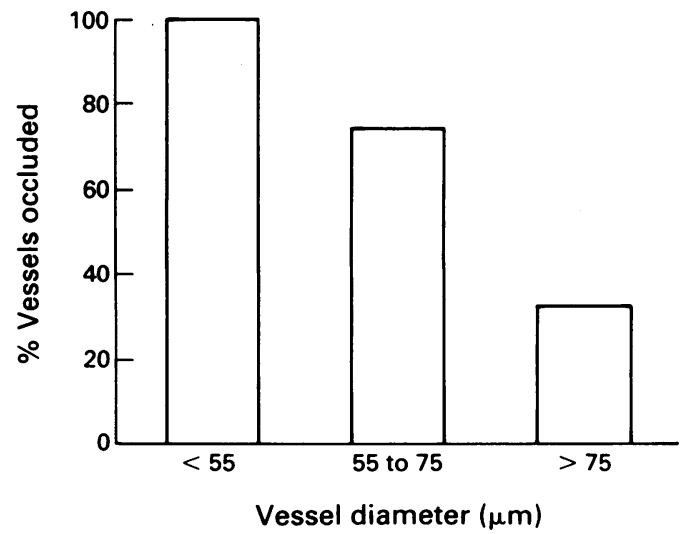

Figure 4 Success rate of vessel occlusion at 2 weeks as a function of the size of the vessel treated. The vessels were treated with 75 and $100 \mathrm{~mW}$. 


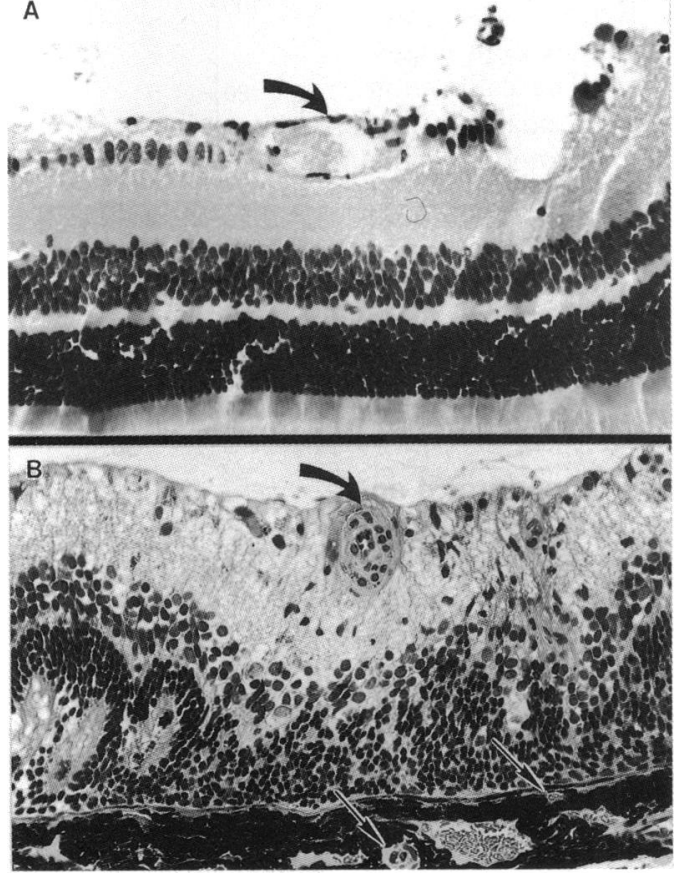

Figure 5 Light photomicrographs of control lesions after treatment. (A) Immediately after the treatment. The retinal vessels (curved arrow) showed thrombi, but the lumina of the blood vessels were still partially patent. (B) Two weeks after the treatment. The treated retinal vessels were reperfused (curved arrow) without thrombi though the wall of vessels were thickened. The choriocapillaris and deeper choroidal vessels were partially recanalised (arrows). There was focal loss of photoreceptor cells while the inner retinal layers were relatively well preserved ( $\times 190$, haematoxylin and eosin).

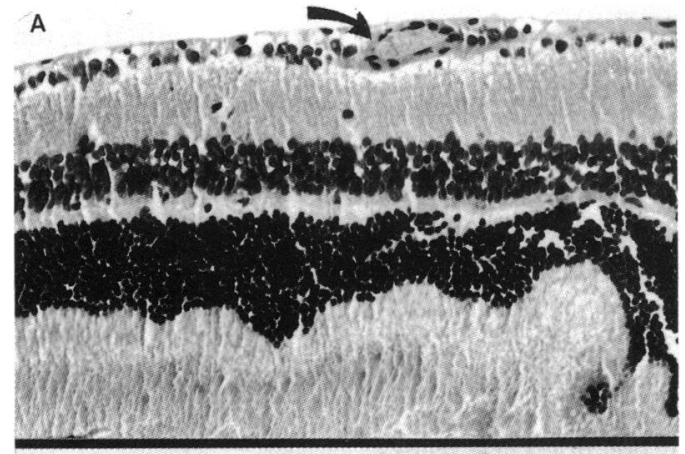

B

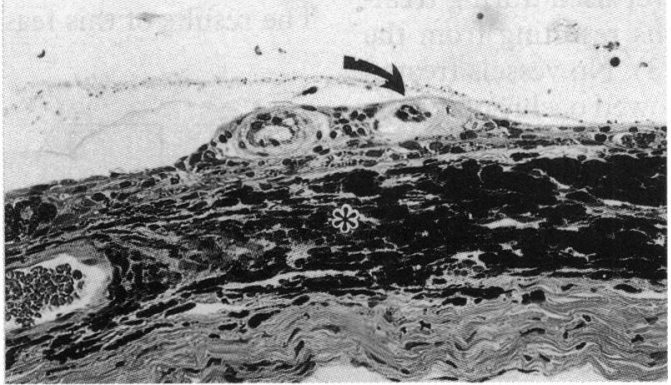

Figure 6 Light photomicrographs of ADP-treated lesions after treatment. (A) Immediately after the treatment. The targeted retinal vessels (curved arrow) were occluded by thrombi consisting of degenerated erythrocytes, platelets, and polymorphonuclear leucocytes. (B) Two weeks after the treatment. The lumina of targeted retinal arteries (curved arrow) became narrow and occluded by thrombi consisting of fibrinous eosinophilic materials. In the choroid (asterisk), the choriocapillaris and deeper choroidal vessels had disappeared. The retina was thinned markedly with extensive loss of neuronal elements in full thickness of the retina possibly due to severe ischaemia ( $\times 190$, haematoxylin and eosin). laser targeted delivery is a potentially valuable method for local delivery of platelet aggregating agents to occlude blood vessels. Targeted delivery was shown to be effective to achieve long term occlusion of retinal arteries. Reperfusion after 2 weeks of control vessels treated only with the laser was consistent with previous studies, ${ }^{56}$ and indicated that the occlusion was caused by the release of ADP. The study has thus illustrated the feasibility of targeting an agent capable of occluding vessels. However, the specific agent used here, ADP, was only a model which is not practical for human use as it can cause transient occlusion of pulmonary capillaries when injected systemically. ${ }^{19}$

The results from the rat studies further characterised the system by indicating that the vascular occlusion by ADP depended on the laser energy and the size of the vessels. The increased effectiveness of treatment at high power (Fig 3) is probably an indication that the effect of ADP needs to be supplemented by thermal damage to the vascular endothelium. This is in agreement with the findings of others. ${ }^{20}$

Histopathological studies revealed thermal damage in the outer retina of the rats but, interestingly, the inner retinal structure was preserved relatively intact. The blue laser is mostly absorbed in the retinal pigment epithelium and the choroid and thus causes a higher temperature than in the retinal vessels. ${ }^{21}$ This effect could be used exclusively to occlude subretinal or choroidal vessels. This could be achieved by restricting the power delivered to the pigment epithelium to that necessary for release in the adjacent vessels without providing enough heat for the release of liposomes in the more remote retinal vessels. On the other hand, by matching the shape of the laser beam to that of targeted retinal vessels, one could cause release in the retinal vasculature while minimising it in the adjacent choroid.

That smaller vessels were more easily occluded than larger vessels is in line with previous studies. ${ }^{22}$ This is because of a combination of effects: (1) platelet aggregates experience less shear in smaller vessels and thus form more easily; (2) the occlusion sustains less pressure; (3) the released ADP is less diluted owing to the smaller flow; (4) ADP released at the targeted site is carried downstream by the blood flow, thereby causing intravascular thrombi distal to the targeted site. (This may be one of the reasons of occlusion of downstream branching arteries despite the reperfusion of treated main artery observed in some rabbits.)

The locally induced occlusion or disappearance of choroidal vessels beneath the retinal lesions treated with ADP liposomes is very promising. As explained above, it is possible to heat the choroid and pigment epithelium to $41^{\circ} \mathrm{C}$, enough to release the drug, without warming the retina excessively. This goal could be achieved if ADP was replaced by a more efficient drug which would not necessitate an accompanying thermal damage. It would then be feasible to occlude subfoveal vascular membranes directly and it is conceivable that the risk to vision would be lower than that of parafoveal photocoagulation. 
Laser targeted delivery of a platelet aggregating agent thus seems to deserve further investigation as a new therapy for a number of ocular diseases such as retinal or subretinal neovascularisation and vascular-rich choroidal tumours. The systemic reaction of the liposomal preparation should be extensively studied before its clinical use, but the fact that liposomes have been safely administered to humans ${ }^{23-25}$ indicates that their use is feasible.

This investigation was supported in part by an International Research Scholar Award (to YO); an unrestricted research grant from Research to Prevent Blindness, Inc, New York; Ophthalmic from Research to Prevent Blindness, Inc, New York; Ophthalmic Research Center Core Grant EY 1792; Grant EY 7768 (to RZ)
from the National Eye Institutes, Bethesda, MD; University from the National Eye Institutes, Bethesda, MD; University
Scholar Award (to RZ) from the University of Illinois at Chicago. Scholar Award (to RZ) from the University of Illinois at Chicago.
The authors have no proprietary interest in the development or The authors have no proprietary interest in the development or
marketing of this methodology. RZ holds a patent for the targeted marketing of this

delivery system. Williams provided photographic services, and Lisa Schoeneman executed the art work.

1 Linner E. Occlusion of the retinal veins in rabbits induced by light coagulation. Acta Ophthalmol (Kbh) 1961; 39: 739-40

2 Hamilton AM, Kohner EM, Rosen D, Bird AC, Dollery CT. Experimental retinal branch vein occlusion in rhesus monkey. I. Clinical appearances. Br f Ophthalmol 1979; 63: 377-87.

3 Becker B, Post LT. Retinal vein occlusion. Clinical and experimental observation. Am f Ophthalmol 1951; 5: 67786.

4 Ferencz JR, Nussbaum JJ, Chopp M, Fisher LJ. Photodynamic therapy for choroidal neovascularization in a new, non-primate model. Invest Ophthalmol Vis Sci (Suppl) 1990; 31: 462 (abstract).

5 Nanda SK, Hatchell DL, Tiedeman JS, Dutton JJ, Hatchell MC, McAdoo T. A new method for vascular occlusion: photochemical initiation of thrombosis. Arch Ophthalmol 1987; 105: 1121-4.

6 Royster AJ, Nanda SK, Hatchell DL, Tiedeman JS, Dutton JJ, Hatchell MC. Photochemical initiation of thrombosis Arch Ophthalmol 1988; 106: 1608-14.

7 Panagopoulos JA, Svitra PP, Puliafito CA, Gragoudas ES. Photodynamic therapy for experimental intraocula melanoma using chloroaluminium sulfonated phthalocyanine. Arch Ophthalmol 1989; 107: 886-90.

8 Kilman GH, Stern D, Gregory WA, Borirak S, Puliafito CA Angiography and photodynamic therapy of experimental choroidal neovascularization using phthalocyanine dye. Invest Ophthalmol Vis Sci (Suppl) 1990; 31: 371.

9 Zeimer RC, Khoobehi B, Niesman MR, Magin RL. A potential method for local drug and dye delivery in the ocular vasculature. Invest Ophthalmol Vis Sci 1988; 7: 1179-83.

10 Zeimer RC, Khoobehi B, Peyman G, Niesman MR, Magin RL. Feasibility of blood flow measurement by externally controlled dye delivery. Invest Ophthalmol Vis Sci 1989; 30: controll.

11 Zeimer RC, Guran T, Shahidi M, Mori MT. Visualization of the retinal microvasculature by targeted dye delivery. Invest the retinal microvasculature by targeted

12 Ogura Y, Guran T, Shahidi M, Mori M, Zeimer R. Feasibility of targeted drug delivery to selective areas of the retina. of targeted drug delivery to selective areas
Invest Ophthalmol Vis Sci 1991; 32:2351-6.

13 Schroder S, Schmid-Schonbein GW, Schmid-Schonbein H, Brab M, Reim M. [Methode zur Erfassung der Netzwerk topologie der menschlichen Retinagefasse.] Klin Monatsb Augenheilkd 1990; 197: 33-9.

14 Gaarder A, Jonsen J, Laland S, Hellem A, Owren PA Adenosine diphosphate in red cells as a factor in the adhesiveness of human blood platelets. Nature 1961; 192: 531-2.

15 Born GVR. Aggregation of blood platelets by adenosine diphosphate and its reversal. Nature 1962; 194: 927-9.

16 Honour AJ, Mitchell JRA. Effect on antibody production of injection of an al-protein fraction. Nature 1963; 197: 101920.

17 Born GVR, Cross MJ. The aggregation of blood platelets. f Physiol 1963; 168: 178-95.

18 Guran T, Zeimer RC, Shahidi M, Mori M. Quantitative analysis of retinal hemodynamics using targeted dye delivery. Invest Ophthalmol Vis Sci 1990; 31: 2300-6.

19 Nordoy A, Chandler AB. Platelet thrombosis induced by adenosine diphosphate in the rat. Scand $\mathcal{F}$ Haemat 1964; 1 : 16-25.

20 Jorgensen L, Hovig T, Rowsell HC, Mustard JF. Adenosine diphosphate-induced platelet aggregation and vascular

21 Bebie H, Fankhauser F, Lotmar W, Roulier A. Theoretica estimate of the temperature within irradiated retinal vessels. Acta Ophthalmol (Kbh) 1974; 52: 13-36.

22 Seiffge D, Kremer E. Influence of ADP, blood flow velocity, and vessel diameter on the laser-induced thrombus formation. Thromb Res 1986; 42: 331-41.

23 Lopez-Berestein G, Bodey GP, Frankel S, Mehta K Treatment of hepatosplenic candidiasis with liposomalTreatment of hepatosplenic candidiasis with

24 Sculier JP, Coune A, Meunier F, Brassinne C, Laduron C, Hooaert C, et al. Pilot study of amphotericin B entrapped in sonicated liposomes in cancer patients with fungal infections. Eur f Cancer Clin Oncol 1988; 24: 527-38.

25 Perez-Soler R, Lautersztain J, Stephens LC, Wright $K$ Khokhar AR. Preclinical toxicity and pharmacology of liposome-entrapped cis-bis-neodecanoato-trans-R, R-1, 2-diaminocyclohexane platinum: II. Cancer Chemother Pharmacol 1989; 24: 1-8. 